\title{
Comment on "Proposed molecular test of local hidden-variables theories"
}

\author{
Emilio Santos \\ Departamento de Física Teórica, Universidad de Santander, Santander, Spain
}

(Received 10 May 1983)

\begin{abstract}
Lo and Shimony have proposed a molecular test of local hidden-variables theories, which should eliminate a loophole arising in all tests dealing with photons. It is pointed out that the proposed correction for accidental coincidences invalidates the test. Conditions for the reliability of the experiment are stated.
\end{abstract}

Lo and Shimony ${ }^{1}$ have proposed an experiment which should allow the refutation of local hidden-variables theories (LHVT) without a loophole arising in the analysis of experiments of polarization correlations of photons. ${ }^{2,3}$ Their proposal is to measure spin correlations of two $\mathrm{Na}$ atoms coming from the dissociation of a $\mathrm{Na}_{2}$ molecule in a singlet state.

The authors derive the following inequality, valid for any LHVT:

$$
\begin{aligned}
S\left(a, a^{\prime}, b, b^{\prime}\right)= & \bar{P}^{++}(a, b)+\bar{P}^{--}(a, b)-[\eta /(2 \eta-1)]\left[\bar{P}^{++}\left(a, b^{\prime}\right)+\bar{P}^{--}\left(a, b^{\prime}\right)\right] \\
& +\bar{P}^{++}\left(a^{\prime}, b\right)+\bar{P}^{--}\left(a^{\prime}, b\right)+\bar{P}^{++}\left(a^{\prime}, b^{\prime}\right)+\bar{P}^{--}\left(a^{\prime}, b^{\prime}\right) \leqslant 2 \eta /(2 \eta-1),
\end{aligned}
$$

where $\eta$ is the efficiency of the atom detectors, and $\bar{P}^{++}$is the probability of detecting a pair of atoms in the + channels of both analyzers conditional upon its being detected at all, and similarly for $\bar{P}^{--}, \bar{P}^{+-}$, and $\bar{P}^{-+}$. The conditional probabilities are related with total detection probabilities through

$$
\begin{aligned}
& \bar{P}^{++}(a, b) \\
& \equiv \frac{P^{++}(a, b)}{P^{++}(a, b)+P^{+-}(a, b)+P^{-+}(a, b)+P^{--}(a, b)},
\end{aligned}
$$

and similar relations for $\bar{P}^{+-}, \bar{P}^{-+}$, and $\bar{P}^{--}$

The prediction of quantum mechanics for the proposed experiment is, for some choice of experimental setup, ${ }^{1}$

$$
\left[S\left(a, a^{\prime}, b, b^{\prime}\right)\right]_{\Psi}=2.5306-0.25[\eta /(2 \eta-1)],
$$

which violates (1) provided that

$$
\eta>0.9 \text {. }
$$

Now, Low and Shimony propose to calculate the probabilities $P^{++}(a, b)$, etc. by assuming that they are proportional to the detection rates after a background subtraction, i.e.,

$$
P^{++}(a, b) \propto R^{++}(a, b) \equiv R_{\exp }^{++}(a, b)-R_{\min },
$$

and similarly for $P^{--}$, etc. Here, $R_{\min }$ is the minimum coincidence rate obtained amongst all possible analyzer orientations. It is assumed that $R_{\min }$ is due to accidental coincidences. The purpose of this Comment is to point out that the proposed background subtraction is incorrect.

In the first place, according to (5) the minimum of $P^{++}(a, b)$ is zero by definition, which excludes $a$ priori all LHVT having strictly positive (nonzero) $P^{++}(a, b)$. In a similar way we might refute quantum mechanics by just adding a constant to Eq. (5). In fact, according to quantum mechanics the minimum of $P^{++}(a, b)$ should be zero. This clearly shows that Eq. (5) cannot be used.

It may be suggested the use of one of the two standard methods to make corrections for accidental coincidences, namely, the study of the time-delay spectrum and the calculation from the single counts rate. ${ }^{4}$ The time-delay spectrum method rests upon the assumption that the coincidence rate is independent of the delay between the first and the second counts of an accidental coincidence. The calculation from the single counts rate assumes that the probability of an accidental coincidence is the product of the probabilities of two single counts. (A good agreement has been reported between both procedures. ${ }^{4}$ ) With any one of these methods, the correction again consists of subtracting a background independent of the relative orientation of the analyzers. In the following we show that this procedure is also not adequate.

According to a general methodological principle, if one attempts to test a theory, the full experiment must be interpreted according to that theory. The subtraction of a constant background may be adequate for a test of quantum mechanics but, for a test of LHVT, it is necessary to calculate the probability of an accidental coincidence from an assumption valid for any LHVT. We propose the following one:

$P_{\mathrm{acc}}^{ \pm}(a, b)=\int_{\Lambda} d \lambda \int_{\Lambda^{\prime}} d \lambda^{\prime} \rho\left(\lambda, \lambda^{\prime}\right) P_{1}^{ \pm}(a, \lambda) P_{2}^{ \pm}\left(b, \lambda^{\prime}\right)$,

which parallels the expression for the probability of a true coincidence [Eq. (3c) of Ref. 1]. Here, we have two different parameters, $\lambda, \lambda^{\prime}$, corresponding to two different $\mathrm{Na}_{2}$ molecules and $\rho\left(\lambda, \lambda^{\prime}\right)$ is the joint probability distribution of these parameters. (For the sake of clarity, a slight change in notation has been made with respect to Ref. 1.)

It can be realized that the standard methods for calculating the probability of an accidental coincidence are equivalent to Eq. (6) plus the assumption

$$
\rho\left(\lambda, \lambda^{\prime}\right)=f(\lambda) f\left(\lambda^{\prime}\right) .
$$

In fact, (7) means that the two $\mathrm{Na}_{2}$ molecules giving rise to an accidental coincidence dissociated in an uncorrelated manner, so that the probability (6) becomes the product of two probabilities for single counts. This is quite plausible for accidental coincidences in a delayed channel. Indeed, the agreement found between the accidental coincidence rate calculated from the single counts rate and the coincidence rate in a delayed channel can be explained as a consequence of the validity of Eqs. (6) and (7) for delayed coincidences. 
In sharp contrast, molecules dissociated at the same (or near the same) time may have correlated hidden variables, $\lambda, \lambda^{\prime}$. Therefore, Eq. (7) may not be valid and the standard methods of background subtraction are incorrect. The maximum possible correlation is obtained if we write, instead of (7),

$$
\rho\left(\lambda, \lambda^{\prime}\right)=f(\lambda) \delta\left(\lambda-\lambda^{\prime}\right),
$$

where $\delta()$ is the Dirac delta. In this case, the accidental coincidence probability has the same dependence on $a$ and $b$ as the true (i.e., nonaccidental) coincidence probability. [However, the rate averaged over $a$ and $b$ may coincide with the one obtained from (7).] Then, the correction should no more consist of subtracting a constant (independent on $a$ and $b$ ) background, but rather in multiplying the experimental rate times a factor between 0 and 1 . Other choices somewhat intermediate between (7) and (8) are also possible, and it is not easy to find the adequate method of making the correction for accidental coincidences.

It might be assumed that a test of the validity of Eq. (7) - and, therefore, the correctness of performing a correction by background subtraction-could be made by varying the dissociation rate (e.g., by a variation of the laser intensity). In fact, Eq. (7) leads to specific predictions about the statistics of coincidences when the dissociation rate changes. (For instance, the rate of accidental coincidences would remain independent of $a$ and $b$, and proportional to the square of the single rate.) However, the confirmation of these predictions is not an absolute proof of the validity of (7). Indeed, it is enough to assume that the functions $P_{1}(\lambda, a)$ and $P_{2}(\lambda, b)$ of Eq. (6) [and Eq. (3a) of Ref. 1] depend on the dissociation rate, in order for the reproduction of any empirical rates to be possible, except if the Bell inequalities are violated even with the uncorrected rates. ${ }^{5}$ It must be stressed that the commented experiment will not be very useful if it needs an untested hypothesis in its analysis because, in these conditions, numerous refutations of LHVT have been reported..$^{3,4}$

The conclusion of our analysis is that Eq. (5) may be valid only for the calculation of the probabilities used in the test of quantum mechanics. In contrast, for the test of the entire family of LHVT, the following relation should be used:

$$
P^{++}(a, b) \propto R_{\exp }^{++}(a, b), \text { etc. }
$$

As a consequence, the function $S$ of (1) cannot be identified with that of (3), but both can be written in terms of $R_{\exp }^{++}, R_{\exp }^{+-}, R_{\exp }^{-+}, R_{\exp }^{--}$, and $R_{\text {min. }}$. Alternatively, we may write (1) and (3) in terms of $R_{\min }$ and the reduced rates $R^{++}, R^{+-}, R^{-+}$, and $R^{--}$, defined by the second Eq. (5).

Choosing the last procedure, Eq. (1) becomes

$$
\begin{array}{r}
\bar{R}^{++}(a, b)+\bar{R}^{--}(a, b)-[\eta /(2 \eta-1)]\left[\bar{R}^{++}\left(a, b^{\prime}\right)+\bar{R}^{--}\left(a, b^{\prime}\right)\right]+\bar{R}^{++}\left(a^{\prime}, b\right)+\bar{R}^{--}\left(a^{\prime}, b\right) \\
+\bar{R}^{++}\left(a^{\prime}, b^{\prime}\right)+\bar{R}^{--}\left(a^{\prime}, b^{\prime}\right) \leqslant[2 \eta /(2 \eta-1)](1+5 \gamma / 4)-3 \gamma / 2,
\end{array}
$$

where

$$
\bar{R}^{++}(a, b)=R^{++}(a, b) /\left[R^{++}(a, b)+R^{+-}(a, b)+R^{-+}(a, b)+R^{--}(a, b)\right]
$$

and

$$
\gamma \equiv 4 R_{\min } /\left[R^{++}(a, b)+R^{+-}(a, b)+R^{-+}(a, b)+R^{--}(a, b)\right] .
$$

For simplicity we assume that $\gamma$ is independent of $a$ and $b$. Now, Eq. (10) can be compared with the quantum predictions (3) and (5), which show that a violation of (10) is possible only if

$$
\eta>(5.06+3 \gamma) /(5.62+\gamma) \simeq 0.9+0.37 \gamma \text {. }
$$

From this inequality and (12) we obtain

$$
R_{\min }<\frac{1}{18}\left[R_{\exp }^{++}(a, b)+R_{\exp }^{+-}(a, b)+R_{\exp }^{-+}(a, b)+R_{\exp }^{--}(a, b)\right]
$$

as a necessary condition for a reliable test of LHVT through the experiment proposed by Lo and Shimony. ${ }^{1}$

It is important to point out that no experiment performed till now have refuted the full family of LHVT. In particular, the atomic-cascade experimental tests have been strongly criticized. ${ }^{6}$ As a consequence, a test along the lines proposed by Lo and Shimony ${ }^{1}$ should be very welcome, if it could be made reliable.

${ }^{1}$ T. K. Lo and A. Shimony, Phys. Rev. A 23, 3003 (1981).

${ }^{2}$ F. Clauser and M. A. Horne, Phys. Rev. D 10, 526 (1974).

${ }^{3}$ See F. Clauser and A. Shimony, Rep. Prog. Phys. 41, 1881 (1978) for a review of LHVT tests.

${ }^{4}$ See, e.g., A. Aspect, P. Grangier, and G. Roger, Phys. Rev. Lett. 47, 460 (1981); 49, 91 (1982).
${ }^{5}$ A. Fine, Phys. Rev. Lett. 48, 291 (1982). In this paper it is shown that, for any quantum correlation experiment which does not violate the Bell inequalities, there exists a local hidden-variables model compatible with the data.

${ }^{6}$ T. W. Marshall, E. Santos, and F. Selleri, Phys. Lett. 98A, 5 (1983). 